\title{
Architettura e vicende costruttive della Rocca di Capalbio (GR): un modello di torrione quattrocentesco ai confini della Repubblica senese
}

\author{
Architecture and construction events of the fortress of Capalbio (GR): a fifteenth \\ century tower model on the borders of the Republic of Siena
}

\author{
Bruno Mussari \\ Università degli Studi Mediterranea di Reggio Calabria, Reggio Calabria, Italy, bruno.mussari@unirc.it
}

\begin{abstract}
Capalbio (GR) is located in the heart of the southern Maremma, along the border strip that in the second half of the fifteenth century marked the line between the Republic of Siena -became part of the Grand Duchy of Tuscany with the peace of Cateau-Cambrésis of 1559- and the Papal State. The historic center, built around the hill on which it stands, enclosed by a double circle of walls, emerges in the skyline of the surrounding landscape. The fortified structure of Capalbio has a non-simple construction history, especially for the remote phases, but that gradually becomes simpler from the second half of the sixteenth century. The reasons why the defence structure was built were exhausted in a relatively short period of time. The advent of firearms and the evolution of the tools and techniques to which the art of war used, as is well known, imposed a radical transformation of military architecture, which only in some cases, responding to a necessarily changed strategy, they were updated or completely renewed. The fortress of Capalbio was not part of the renovation program and this decision allowed the Maremma village to maintain its historic medieval core until the modern era. The results of this research derive from the identification and study of fifteenth century construction accounting documents, compared with the structures that still exist. It was thus possible to retrace the main construction and transformation phases of the fortified complex, identifying the period in which it was built. Finally, it is not by chance that in that context the fortress of the Rocca replicates a reiterated model, probably due to the widespread use of Ticino and itinerant Lombard workers, also documented on this site.
\end{abstract}

Keywords: Capalbio, Republic of Siena, tower, fortress, Lombard workers.

\section{Introduzione}

Capalbio è posta "in un Poggio assai elevato, che dalla parte di Mezzogiorno scuovre il Mare Toscano e quasi tutta la spiaggia Romana", al confine tra la Repubblica di Siena e lo Stato della Chiesa. Le storia della fortificazione di Capalbio, complessa in epoche remote, si dissipa dalla seconda metà del XVI secolo con la caduta della Repubblica senese nel 1559, la sua inclusione nel Granducato e il riassetto del territorio (Ascheri,
2001). Sotto i Medici il ruolo difensivo di Capalbio si esaurì in breve tempo: l'evoluzione degli strumenti e delle tecniche dell'arte della guerra imposero una radicale trasformazione delle fortezze, cui solo alcune vennero destinate. Capalbio rimase esclusa, e se "avanti l'uso della polvere si sarebbe giudicata inespugnabile per l'altezza e grossezza delle muraglie"2, avrebbe a breve perso l'originaria inviolabilità, conservando però la 


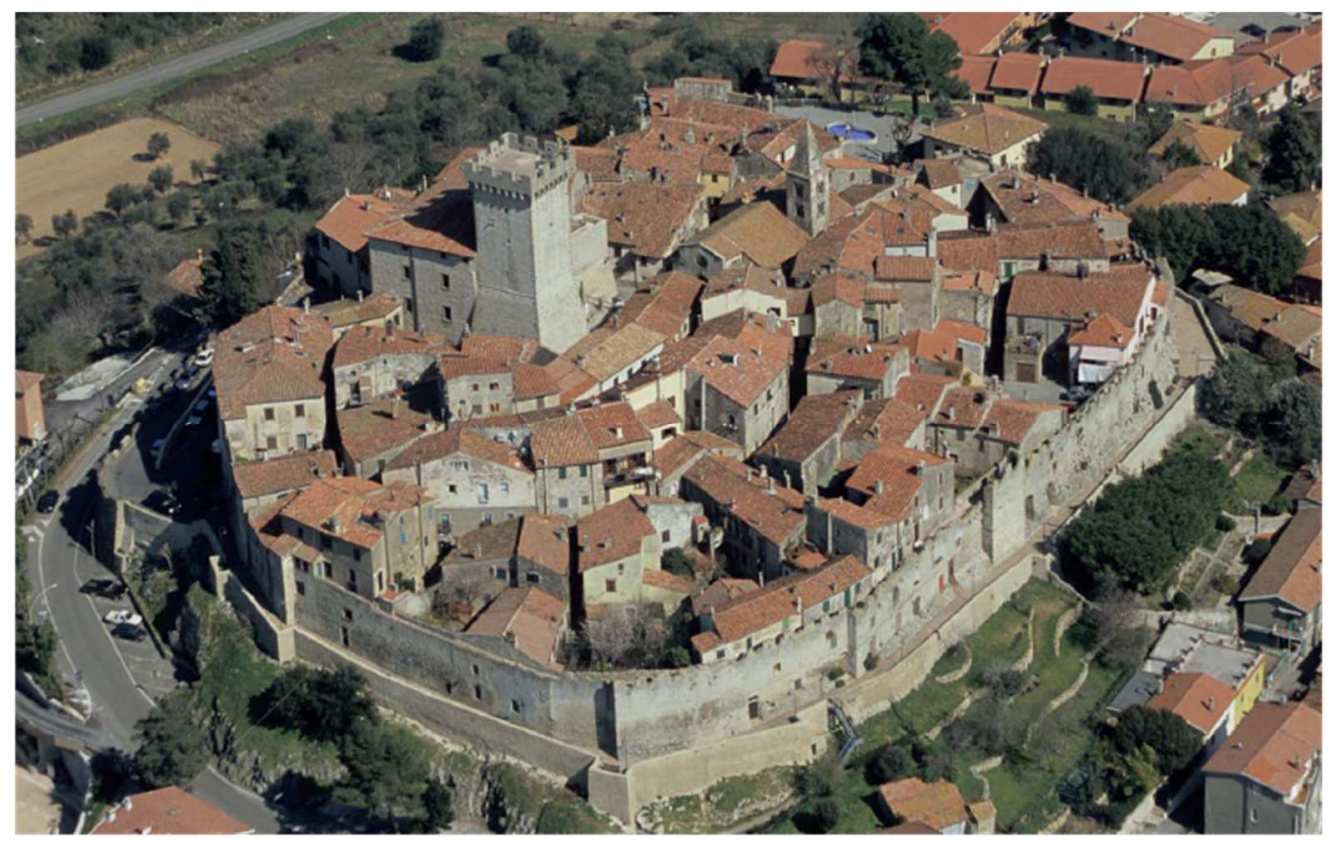

Fig. 1. Capalbio (GR), veduta aerea del nucleo storico cinto dalle mura. Sulla sommità emerge la Rocca con la torre.

memoria del nucleo storico medievale, caratterizzatosi nel passaggio da quell'era all'età moderna (Fig. 1).

\section{La fortificazione}

Capalbio dovrebbe risalire alla fase di formazione di villaggi fortificati e all'incastellamento avvenuto tra XI e XIII secolo, come attesterebbe il modello insediativo concentrico circoscritto dalle mura, imperniato sulla rocca, molto diffuso in Maremma (Farinelli 2007). Il primo documento in cui si nomina Capalbio risale al 1161, nel privilegio emesso da Alessandro III (1159-1181) a favore del Monastero di S. Anastasio ad Acquas Salvias - che possedeva il distretto della città di Ansedonia con il castrum Capalbi- attraverso il quale la Santa Sede esercitava il controllo sull'Argentario e sulla Maremma meridionale, contenendo la progressiva affermazione degli Aldobrandeschi, al cui contado miravano Orvieto e Siena (Angelucci, Bellettini, 2006; Domenichelli, 2010). É nella fase comitale e aldobrandesca che si assiste alla fortificazione di molti siti posti su nodi di rilevanza strategica in quel territorio. Anche se per Capalbio le fonti non documentano la realizzazione di strutture difensive, nei carteggi del XIII e XIV secolo il centro maremmano è a volte denominato castrum $^{3}$, cioè centro abitato munito di cinta muraria, come documenti dell'epoca lasciano chiaramente presumere (Mussari, 2012). La sua cinta muraria, nonostante le modifiche intervenute che ne hanno nel tempo alterato lo stato, conserva la matrice originaria; alte mura merlate di pietrame dallo spessore contenuto, intervallate da torrette rettangolari poco sporgenti, mosse da riseghe per le archibugiere per il tiro di fiancheggiamento. Le arciere erano in genere ricavate nei merli, sui quali si attestano ancora le mensole lapidee per il sostegno delle ventiere o mantellette, i ripari mobili lignei destinati a coprire i soldati in movimento lungo il camminamento di ronda. Il loro discreto stato di conservazione è anche in parte attribuibile al fatto che gran parte degli interventi effettuati in seguito, di cui rimangono tracce evidenti, vennero eseguiti nel corso del XV secolo (Mussari, 2012), nell' ambito di una tradizione costruttiva e tipologica consolidata e nella permanenza di tecniche e strumenti di difesa che non ne hanno stravolto il carattere (Settia, 2003). La configurazione della fortificazione di Capalbio e la costruzione della Rocca avvenne con il dominio senese. 
Il primo settembre 1416 la comunità fu conquistata a nome di Siena da Ranuccio Farnese, succeduto agli Aldobrandeschi, nel corso della guerra di Pitigliano contro Bertoldo Orsini. Per accerchiare Pitigliano e Sorano, con l'intento di isolarle e costringerle alla resa, vennero conquistante alcune rocche limitrofe: "Le rocchette le prese le genti de Sanesi el Casaro adì 26 e poi ebero altrichosto e poi ebero chaparbio adì primo de settembre e poi a dì 4 ebero manciano questi fu l'anno 1416" ". Con queste imprese Siena consolidava il proposito maturato già nel secolo precedente di estendersi verso il mare, avendo la meglio su Orvieto e Pisa. Nei Capitoli stilati tra la Comunità conquistata e la Repubblica di Siena ${ }^{5}$, come di consuetudine, uno era dedicato alla fortificazione della città, sancendo "que custodia arcis sive cassari dicti castri sit et esse debet in perpetuum in fortia dicti comunis Senarum et pro ipsum comune senarum debeat fieri sicut ei pla-

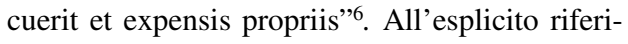
mento alla gestione della Rocca non corrisponde quello per la custodia delle mura, per le quali, in ogni caso, anche Capalbio beneficiò del fondamentale supporto senese (Mussari, 2012). Sin dal settembre del 1416 venne nominato il castellano ${ }^{7}$, e nel successivo mese di ottobre Siena inviò maestro Lorenzo "ad approvidendum casserum caparbii" $"$ e i primi strumenti di difesa9. Alla fine di febbraio del 1417 fu stipulato l'accordo con i maestri, locali e lombardi, che dovevano costruire la Rocca con la torre, sulla quale concentriamo l'attenzione: "Et visa et intelecta locatio facta pro comissarios concistorii ut patet mani mei notarii de Castellonatu Capalbii et de Muraglia et edifitio fiendo in dicto cassaro facta Nanni Grasselli de Senis et Magistro Johanni de Como et Magistro francisco magistri Johannis et Magistro Aliotto lombardis"10. I lavori sarebbero durati oltre due anni, come attesta la verifica sulle opere effettuata il 20 ottobre $1419^{11}$. Anche se non sono stati rintracciati i capitoli dell'accordo con i costruttori rimasti al notaio- la descrizione dettagliata dei lavori, che giustificava la corresponsione dei compensi alle maestranze coinvolte, consente di ripercorrere la costruzione della Rocca e di affermare che la torre e buona parte del circuito murario che circoscrive la piazza interna del cassero sono dell'inizio del XV secolo e non della fase aldobrandesca come si era creduto. Il "palazzo", inglobato nella rocca e corredato degli apparati che lo armonizzavano alla fortezza rinnovata, invece, esisteva già nel 1419 (Mussari, 2012).

\subsection{La Rocca}

Il documento quattrocentesco che dimostra la paternità senese della Rocca di Capalbio fu redatto da pietro dagnolo dellabaco ${ }^{12}$. L'analisi della fabbrica procede dall'esterno verso l'interno, attraverso l'antiporta, passando per il palazzo, raggiungendo il 'chiostro' o cortile, dedicando infine un'intera partita alla torre maestra. I riferimenti individuati dal revisore per localizzare la posizione delle diverse sezioni in cui venne scissa l'analisi della fabbrica erano: "la chiesa", la chiesa di san Nicola; la "terra", il nucleo abitato; il "palazzo", posto nella parte più alta del borgo; "l'antiporto", lo spazio di passaggio tra l'esterno delle mura e l'abitato. L'antiporto, oggi non immediatamente individuabile, si identifica dalla lettura comparata tra il documento senese e la mappa del centro abitato riprodotta nella tavola di Antonio Maria Bartolini nel 1763 e nel catasto Leopoldino (Fig. 2).

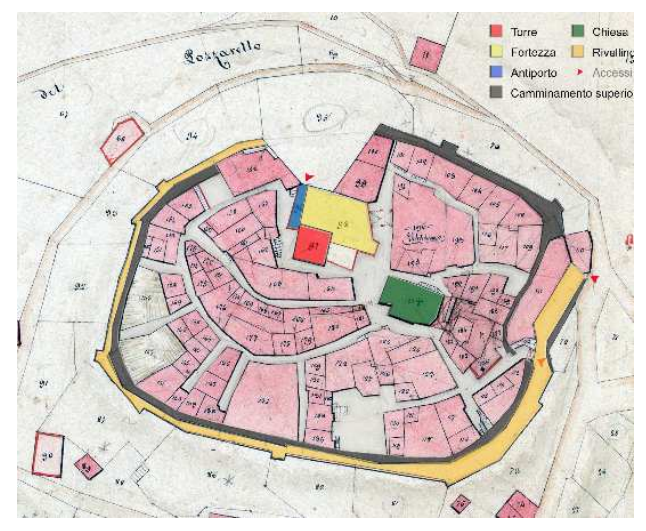

Fig. 2. Capalbio, Catasto Leopoldino rielaborato. In evidenza le strutture difensive principali oltre alla chiesa di S. Nicola (da Mussari 2012).

Alla Rocca si accedeva dall' antiporta, all'interno della quale si apriva "la prima porta dentro alanti porto per andare ala porta seconda", posta "fra il palazo e la torre", dalla quale si entrava nel cor- 
tile. Dalla descrizione riportata si ipotizza si trattasse delle porte che delimitavano il passaggio, oggi ridotto, tra la torre e il palazzo originario. Solo per alcuni tratti e per una parte della cortina settentrionale $^{13}$, oltre che per il palazzo del tempo, pietro d'agnolo fa riferimento a strutture preesistenti, il resto è computato come nuova costruzione. Il cortile, dove furono realizzate finestre "a bucha" con relativi ripari -forse le postazioni ricavate nella rimaneggiata cortina meridionale- era prima completamente libero, mancando il corpo di fabbrica sul lato settentrionale costruito successivamente. Il "cortile" era percorso da un "corridoio" continuo lungo tre lati e interrotto da un "torricello [...] nel canto verso la chiesa, tra la cortina che si innesta sulla torre e quella del fronte orientale. La lunghezza dei tre tratti di mura misurati da pietro d'agnolo, che con il "torricello" definiscono il perimetro della piazza interna, corrisponde con buona approssimazione a quello attuale. Le cortine, in origine dotate di "confesso", con basamento a scarpa e redondone, erano concluse da "merli e pettorali", di 4 braccia d'altezza per tre quarti di braccio di spessore, che coronavano anche i due lati lunghi del palazzo e il "torricello"14: una configurazione del complesso fortificato riconducibile alla rappresentazione della città posta a margine della tavola settecentesca di Antonio Maria Bartolini (Fig. 3).

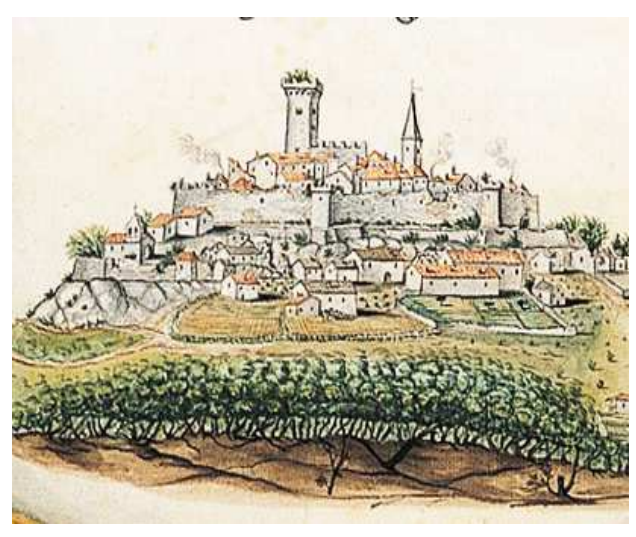

Fig. 3. Veduta di Capalbio presa da Mezzogiorno, Antonio Maria Bartolini, 1763 (ASF, Miscellanea di Piante, 46 - da Mussari 2012).

Il ponte levatoio, cui si accenna alla fine della relazione, in una partita dedicata ai blocchi lapidei per i beccatelli ${ }^{15}$, senza indicare dove si trovasse, doveva connettere la porta soprelevata di accesso alla torre con il palazzo e il camminamento di ronda del circuito murario perimetrale.

\subsection{La torre}

Nella "misura della torre maestra" la corrispondenza tra il documento e i dati metrici verificabili è puntuale. La fabbrica della torre venne scomposta nelle quattro facce correlandole al contesto circostante: la "faccia della torre maestra verso la chiesa" ad est; quella "della detta torre verso la terra" a sud; quella "al entrata della porta del anti porto" o "che mena verso alentrata delantiporto" a ovest; l'ultima "dentro ali due porte" o "dentro ala porta sotto lentrata" o "colla porta della entrata dal bastone"16 a nord, in corrispondenza dell' accesso alla Rocca e al cortile. Nel computo fu eseguita prima la misura delle pareti a scarpa fino al bastone, separando la parte inclinata da quella retrostante, non accennando, però, al nucleo del basamento, per proseguire poi con la sezione della torre in elevazione, fino alla sommità. La valutazione è fatta per ogni "faccia" e per ognuno dei due piani voltati in cui la torre si articola in alzato, il primo più basso, il secondo decisamente più alto. Il minimo scarto rilevabile tra le misure riportate nel documento e quelle verificabili sul rilievo della torre è così esiguo da non generare dubbi sulla sua identificazione (Figg. $4 \mathrm{e}$ $5)$.

La torre era conclusa in sommità dalla serie di beccatelli composti da più blocchi di pietra "macigna" sovrapposti dal profilo a quarto di cerchio: quattro in corrispondenza degli angoli e tre lungo le cortine. Sui beccatelli si impostavano archetti, più ampi quelli angolari, all'interno dei quali erano ricavate le caditoie per la difesa piombante, a sostegno dei parapetti e dei merli con le arciere. Pietro d'agnolo quantificò "32 imposte di beccategli sotto i merli della corona della torre [...] Merli e pettoragli [...] per lunghezza braccia 55 1/2 [...] Archetti sotto i detti merli e pettoragli per altezza braccia $1 \frac{1}{2}$, grossi braccia 1 , longhi braccia $55^{1 / 2}{ }^{\prime \prime 17}$, esattamente nel numero e nello sviluppo lineare verificabile sulla torre di Capalbio: come di consuetudine, infine, in cima alla torre fu costruito un campanile. 

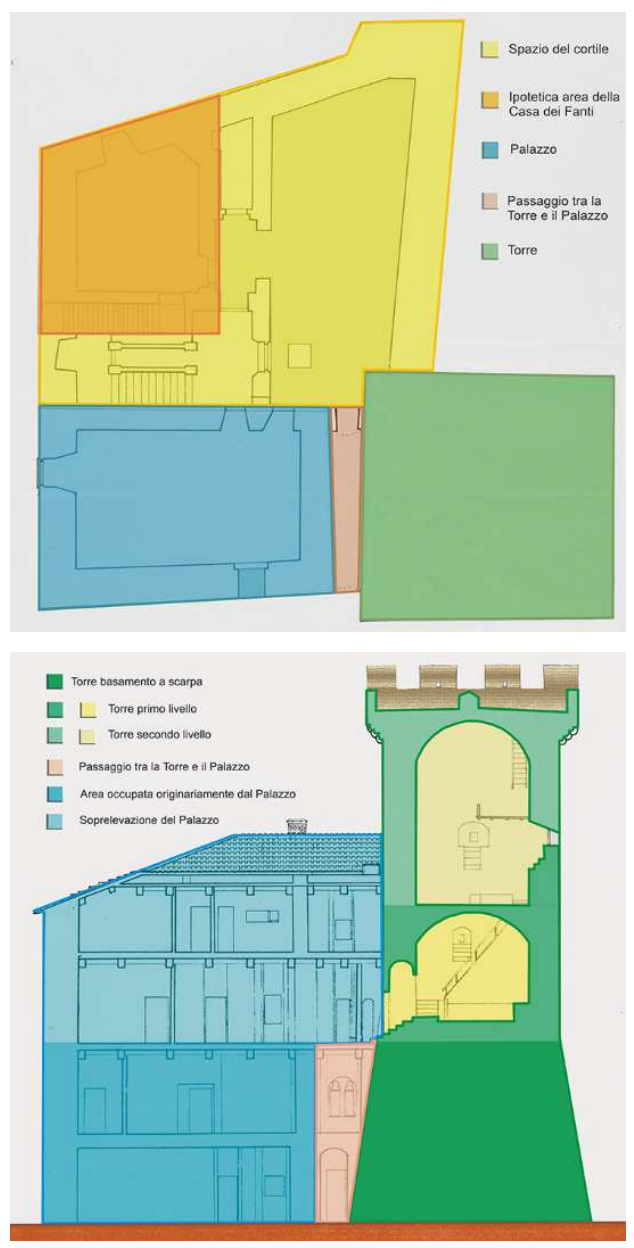

Figg. 4-5. Planimetria e sezione trasversale della Rocca. Le campiture evidenziano le diverse aree che compongono la fortezza (Elaborazione del'autore).

La Rocca era stata edificata nella sua totalità, motivando la parzialità degli interventi successivi. Nei lavori che "Pavolo di ser Giovanni e Lorenzo de Rocchi”, già castellani del cassero di Capalbio, proposero di eseguire con l'istanza al Consiglio Generale del 23 febbraio $1450^{18}$, si prevedeva di costruire in tre anni una casa per i fanti all'interno del cortile -"dal capo della scala che va al palazo infino al muro del circuito desso cassaro"- occupando lo spazio libero sul fronte settentrionale. La costruzione della casa e le opere manutentive nel palazzo e nella torre, approvate dal Consiglio Generale e dal Comune di Siena ${ }^{19}$, vennero verificate alla fine dei tre anni da un maestro esperto lombardo - "magistrus pietrus lombardus muratorem expertum et doctum in similibus"- che, accompagnato da un notaio, si recò sul posto il 6 dicembre 1452 constatando l'effettiva realizzazione dei lavori ${ }^{20}$. La configurazione della rocca, anche con queste ultime opere, era definita. Interventi successivi ne hanno poi mutato l'assetto originario. La torre è stata preservata nella sua quasi totale originalità -nonostante alcuni interventi di restauro-, mentre il circuito murario risulta privo delle merlature e del "torricello". Il palazzo, riconvertito prima in edificio pubblico e poi privato, è stato riconfigurato inglobando la casa dei fanti ed elevato di ulteriori piani, per rispondere alle esigenze delle famiglie che dal XVI secolo lo hanno avuto in concessione (Mussari, 2012; Rotundo, 2012).

\section{Modello e maestranze}

La torre della Rocca quattrocentesca di Capalbio rappresenta un modello diffuso nel dominio senese, un possente mastio quadrangolare con basamento a scarpa concluso da un redondone, sopra il quale si eleva il corpo del torrione concluso dal coronamento merlato sporgente sorretto da archetti pensili su beccatelli, tra i quali si aprivano le caditoie per la difesa piombante (Fig. 6). Il documento non lascia dubbi sul fatto che la torre sia stata interamente realizzata al principio del XV secolo con il concorso di maestranze lombarde, la cui presenza e concentrazione nel dominio senese, specie nella fase di espansione della Repubblica verso sud, rappresenta una singolarità messa già in evidenza anni fa da Cecchini (Cecchini, 1959), e solo in parte analizzata per la sua incidenza nella produzione architettonica, non solo militare, nel contesto senese (Pinto, 1982, 1984; Bianchi 1996). Dal punto di vista tipologico bisogna considerare che nell'ultima fase del medioevo il linguaggio che connota l'architettura militare non subisce significative variazioni, basti ricordare il disegno trecentesco della torre di Montalceto, per constatare la ricorrenza di caratteri che si ritrovano anche nei disegni di Francesco di Giorgio. La torre di Capalbio ripropone i caratteri di un modello diffuso tra le Comunità soggette a Siena nel XV secolo, come il disegno più tardo della rocca di Gavorrano, acquisita nel $1464^{21}$, ulteriormente conferma (Fig. 7). 


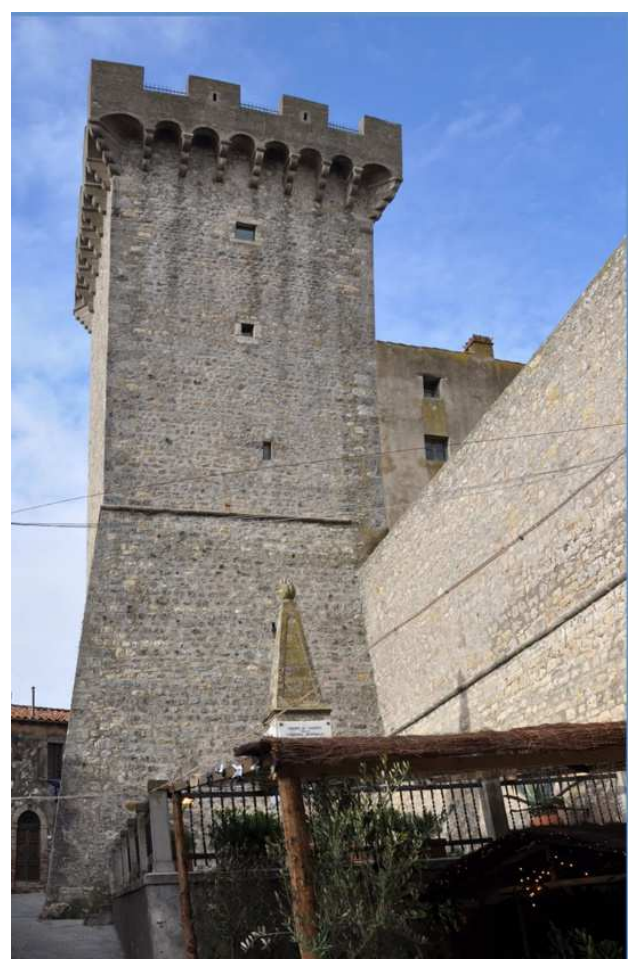

Fig. 6. Capalbio (GR). La torre della Rocca.

Si trattava di un tipo la cui scelta era connessa a tradizioni costruttive consolidate, di cui i maestri muratori lombardi, espressione di un fenomeno migratorio non marginale, erano specialisti esperti e detentori, oltre alla necessità di contenere i tempi di realizzazione nei molteplici cantieri aperti, in cui i costruttori locali erano necessariamente affiancati dai maestri 'dei laghi' (Bianchi, 1996), come i documenti dell' Archivio di Stato di Siena diffusamente confermano. La loro presenza è accertata sin dal XIV secolo, e non solo nel ruolo di semplici lapicidi, nelle fortificazioni di Bagno Vignoni, Sinalunga, Petriolo, Grosseto, Montepulciano, Arcidosso, Lucignano, Talamone, Montalcino, Fighine, Saturnia, San Quirico d'Orcia, Radicofani, Sarteano, per citare alcuni esempi (Figg. 8 e 9).

A convalidare l'idea della riproposizione di un modello concorre inevitabilmente anche la mobilità itinerante di quelle maestranze migrate in Toscana, non è infatti da considerarsi un caso isolato che i libri della Biccherna documentino negli stessi anni la presenza di quasi tutti i maestri impegnati nella costruzione del cassero di Capalbio anche nel cantiere di quello di Radicofani ${ }^{22}$. In un contesto siffatto, infine, non è trascurabile il valore simbolico che a quelle torri maestre si potrebbe attribuire, essendo in qualche modo chiamate a rappresentare la vigile presenza di Siena nel suo contado.

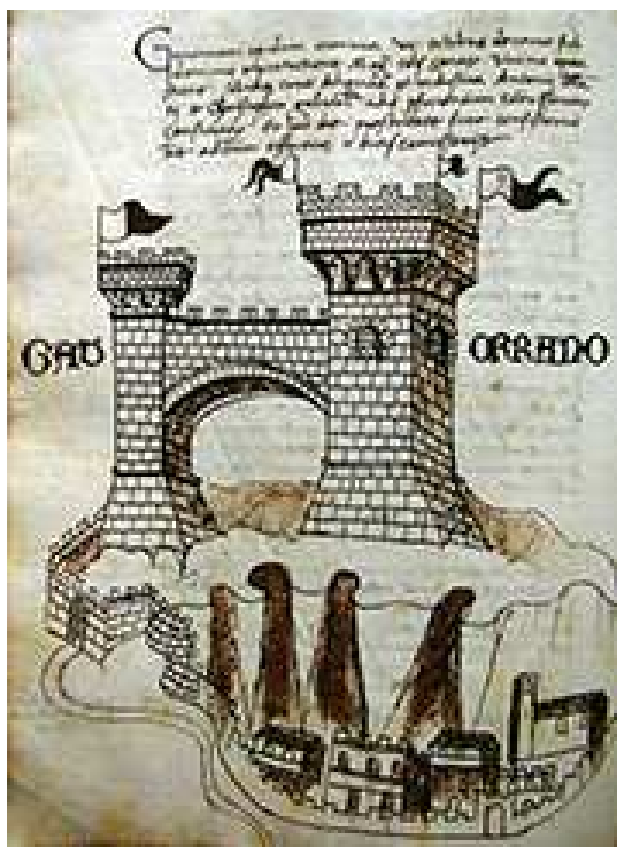

Fig. 7. Gavorrano riproduzione della Rocca, 1464 (ASS, Concistoro, 588, c. 40v - da Mussari 2012).

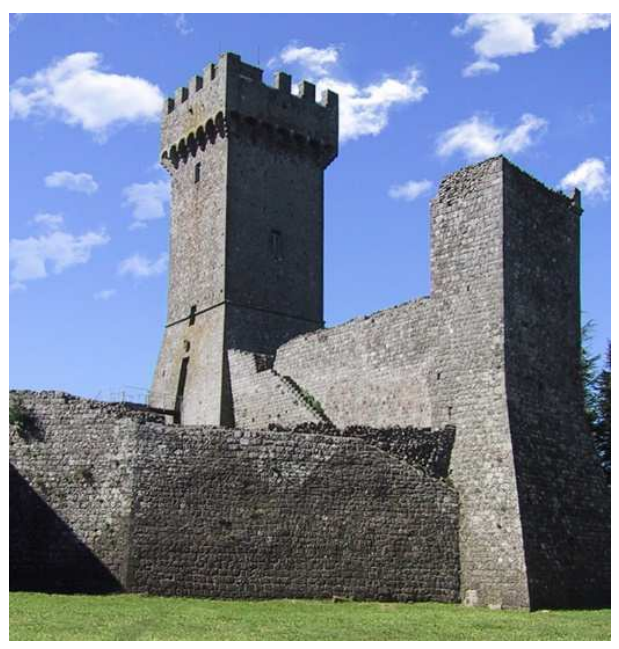

Fig. 8. Radicofani (SI). La Rocca quattrocentesca. 


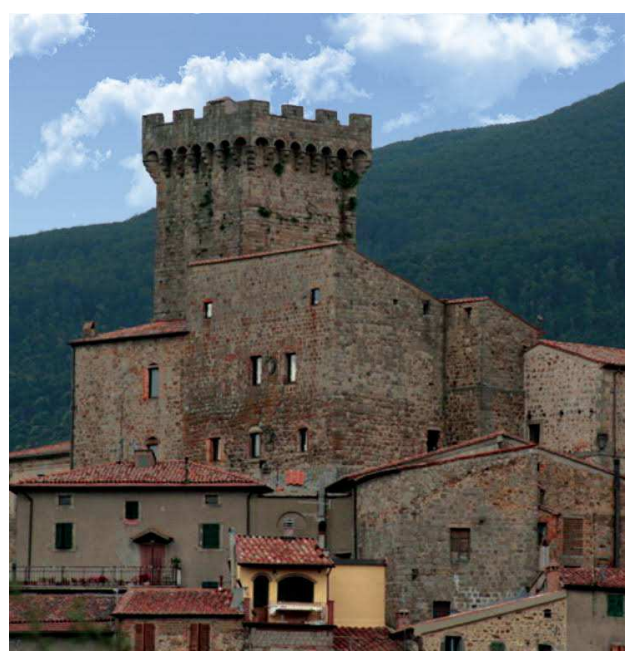

Fig. 9. Arcidosso (GR). La Rocca con la torre.

\section{Note}

1 Archivio di Stato di Siena (ASS), ms. D 68, c.125; Biblioteca Comunale degli Intronati di Siena (BCS), ms. B.IV.9. Per la descrizione del circuito murario ASS, ms D. 84, c. 300.

${ }^{2}$ ASS, ms. D 68, c.125.

${ }^{3}$ ASS, Capitoli 3, Caleffo Nero, c. 206r, 18 agosto 1345; Ivi, c. 234r, 14 novembre 1345.

${ }^{4}$ BCI, ms. A.VII 44, c. 462v. Si veda anche ASS, Concistoro, 304, c. 9v, 7 settembre 1416.

${ }^{5}$ BCI, ms. C. II 5, c. 80v. Si veda anche BCI, ms. B.X.5, c. 100 r.

${ }^{6}$ ASS, Capitoli, 41, c. 101v, 17 settembre 1416;
ASS, Capitoli, 139, copia del 13 ottobre 1519

${ }^{7}$ ASS, Concistoro, 304, c. 9v, 7 settembre 1416.

8. Ivi, c. 46v, 13 ottobre 1416;

9 ASS, Concistoro, 305, c. 12r, 19 novembre 1416; Ivi, c. 13v, 21 novembre 1416.

${ }^{10}$ ASS, Concistoro, 306, cc. $29 v$, 30v, 26 febbraio 1417.

${ }^{11}$ ASS, Regolatori, 12, cc. 218v-222r, 20 ottobre 1419.

12 Ibidem. Da non identificarsi con Pietro di Checco dellabbaco, si veda Adams, 1985, pp. 384-395; Mussari, 2010, pp. 175-211.

${ }^{13} \mathrm{Ivi}$, c. $219 \mathrm{r}$.

${ }^{14} \mathrm{Ivi}$, cc. $219 \mathrm{v}-220 \mathrm{r}$.

${ }^{15} \mathrm{Ivi}$, c. $221 \mathrm{v}$.

${ }^{16}$ Ivi, cc. 220v-221r.

${ }^{17}$ Ivi, cc. 221v-222r.

${ }^{18}$ ASS, Consiglio Generale, 224, cc. 331r-332v, 23 febbraio [1449] 1450.

${ }^{19}$ ASS, Consiglio Generale, 224, c. 332v, 23 febbraio [1449] 1450; ASS, Concistoro, 2462, cc. $83 \mathrm{v}-84 \mathrm{v}, 28$ febbraio [1449] 1450.

${ }^{20}$ ASS, Concistoro, 2462, 24 dicembre 1452, cc. 93r, 93v.

${ }^{21}$ ASS, Concistoro, 588, cc. 40r-40v

${ }^{22}$ ASS, Biccherna, 452, anno 1419, c. 113r; c. $154 \mathrm{v}$; ASS, Biccherna, 453, anno 1420, c. 86r.

\section{Bibliography}

Adams, N. (1985). "The life and times of Pietro dell'Abaco, a Renaissance estimator from Siena (active 1457-1486)", Zeitschrift für Kunstgeschichte, 48, pp. 384-395.

Angelucci, V.; Bellettini, B. (2006). Capalbio. Aspetti della sua storia dal medioevo all'età moderna, Betti, Siena. Ascheri, M. (2001). Lo spazio storico di Siena, Pizzi, Cinisello Balsamo (Mi).

Bianchi, G. (1996). Maestri costruttori lombardi nella Toscana centro-meridionale (secoli XII-XV), Indizi documentari ed evidenze materiali., in Della Torre, S.; Mannoni, T.; Pracchi, V., eds., Magistri d'Europa, Edinodo, Milano, pp. $155-166$.

Francovich, R.; Ginatempo, M., eds. (2000). Castelli. Storia e archeologia del potere nella Toscana medievale, All'insegna del Giglio, Firenze.

Cecchini, G. (1959). "Maestri luganesi e comaschi a Siena nel secolo XV”, in Arslan, E., ed., Arte e artisti dei laghi lombardi, Noseda, Como, pp. 131-150. 
Domenichelli, L. (2010). Capalbio, Laurum, Grosseto.

Farinelli, R. (2007). I castelli nella Toscana delle "città deboli": dinamiche del popolamento e del potere rurale nella Toscana meridionale (secoli VII - XIV), All'insegna del Giglio, Firenze.

Monaci, F. (2010). "Il castello di Arcidosso", in Pellegrini, E., eds., Fortificare con arte. Seconda serie di studi sulle vicende storiche e architettoniche di alcuni castelli dell'antico territorio senese, Pistolesi, Siena, pp. 7-14.

Mussari, B. (2010). "Il castello e la Rocca di Fighine", in Pellegrini, E., ed., Fortificare con arte. Seconda serie di studi sulle vicende storiche e architettoniche di alcuni castelli dell'antico territorio senese, Pistolesi, Siena, pp. 175211.

Mussari, B. (2012). "Tra gli Aldobrandesci e il Granducatio: architettura e vicende costruttive delle fortificazioni di Capalbio", in Mussari, B.; Rotundo, F., eds., Capalbio. Storie di un Castello, Effigi, Arcidosso (GR), pp. 33-72.

Pinto, G. (1982). "L'immigrazione di manodopera nel territorio senese alla metà del Quattrocento”, in La Toscana nel tardo Medioevo, Sansoni, Firenze, pp. 421-449.

Pinto, G. (1984), "L’organizzazione della difesa: i cantieri delle costruzioni militari nel territorio senese (secoli XIV XV)”, in Comba, R.; Settia, A.A., eds., Castelli. Storia e archeologia, Assessorato alla cultura Regione Piemonte, Torino, pp. 259-268.

Pizzoni, S. (2009). "Sarteano: il cantiere quattrocentesco del castello", in Pellegrini, E., ed., Fortificare con arte. Vicende storiche ed architettoniche di quattro castelli senesi, Pistolesi, Siena, pp. 79-116.

Rotundo, F. (2012). "Sviluppo urbano e architettura a Capalbio dal XIII al XX secolo", In Mussari, B.; Rotundo, F., eds., Capalbio. Storie di un Castello. Effigi. Arcidosso (GR). pp. 73-103.

Scarampi, A. (2010). San Quirico d'Orcia e le sue fortificazioni. in E. Pellegrini. a cura di. Fortificare con arte. Seconda serie di studi sulle vicende storiche e architettoniche di alcuni castelli dell'antico territorio senese, Pistolesi, Siena, pp. 51-110.

Settia, A. (2003). "I caratteri edilizi di castelli e palazzi”, in Castelnuovo, E.; Sergi, G., eds., Arte e storia del Medioevo, Del costruire: tecniche, artisti, artigiani e committenti, Einaudi, Torino, vol. II, pp. 187-212. 